(2) Open Access Full Text Article

\title{
Improving the chemotherapy process and service to cancer patients
}

This article was published in the following Dove Press journal:

Risk Management and Healthcare Policy

20 April 20II

Number of times this article has been viewed

\author{
A Garcia-Alonso \\ North Wales Cancer Treatment \\ Centre, Glan Clwyd Hospital, \\ Bodelwyddan, Wales, United Kingdom
}

\begin{abstract}
The North Wales Cancer Treatment Centre (NWCTC) has to deal with an increasing demand in the number of patients who require chemotherapy, with the escalating use of second line, third line, and additional treatment for many cancers. As a result, there is growing pressure on the chemotherapy unit to deliver treatment quickly, efficiently, and safely. Following guidelines from the Department of Health's Manual for Cancer Services, we are constantly looking for ways to improve and develop the level of care provided at our center, and the process of receiving chemotherapy has been identified as an area of high risk. Therefore, a team was established to review and explore current practices at the NWCTC with the goal of implementing an improved process to minimize the risks of chemotherapy treatment.
\end{abstract}

Keywords: chemotherapy, modernization tools, referral forms, chemotherapy day unit

\section{Introduction}

Each day more than one million people are safely and successfully treated at the National Health Service (NHS), but evidence suggests that errors occur in complex health care systems resulting in the risk of harming patients. Once patients enter the system, they have the right to expect that every effort is made to ensure that their care and treatment are both safe and effective. Everyone who works at the NHS contributes to the system that delivers health care, whether they hold a direct clinical or nonclinical position.

Health care systems that continue to use processes that do not work cause delays in the system and result in high levels of error, and it is widely recognized that these processes need radical redesign. Therefore, patient safety is everybody's responsibility. ${ }^{1}$

\section{Background}

The North Wales Cancer Centre opened in 2000, and the chemotherapy day unit now performs an average of 500 cycles of chemotherapy per month. Following discussions at the Divisional Risk Management meeting, it was decided that a group was required to analyze the complexities of the chemotherapy process and ensure that all patients receive treatment specific to their diagnosis and with minimal complications within a reasonable period of time. A professional Chemotherapy Improvement Group was established comprising nurses, pharmacists, social workers, ward sisters, dieticians, consultants, and patients. The Chemotherapy Improvement Group meetings were initially scheduled every two weeks and later every month for a period of 18 months., ${ }^{2,3}$
Correspondence: A Garcia-Alonso North Wales Cancer Treatment Centre, Glan Clwyd Hospital, Bodelwyddan,

Wales, United Kingdom

Tel +44 I745 445738

Fax +441745445212

Email agaralon@hotmail.com 
The aim of these meetings was to work effectively as a team and to understand not just one's own speciality, but to gain insight into other areas of expertise, which would, in turn, help the North Wales Cancer Treatment Centre run more efficiently and effectively.

The group was created to review and explore current practices and to identify areas of risk during the chemotherapy treatment of patients with cancer. The goal was to implement and improve the process for all concerned.

We recognize that the chemotherapy process carries a particularly high degree of risk. Therefore, we established an objective to improve patient care during chemotherapy treatment through the improvement of communication between the clinical areas, pharmacy, nursing staff, and clinical staff.

This analysis was planned as an audit of the process of chemotherapy treatment of cancer patients to provide a clear map of the pathway followed by patients on treatment and to address potential risk factors during the process. At the same time this study is a pioneer for larger studies looking into different issues and insights regarding risk management science in this patient population.

\section{Starting point}

We examined a patient's journey from the original referral through to the first consultation with the consultant clinical oncologist and throughout the rest of the chemotherapy treatment.

The Chemotherapy Improvement Group reviewed the service and targeted specific areas for improvement by adopting a modernization approach. The team chose the tool of process mapping, and several sessions were undertaken to enable the team to dissect a patient's journey by utilizing tools for modernizing services and data collected from complaints from patients, staff, and incident reports.

\section{Process mapping}

The currently existing care process was described. The team analyzed where the problems exist throughout the process and questioned who, where, and in what sequence each step is performed, all the while asking if there was a better way to achieve the same goal.

The exercise aimed to identify the following:

Claims: What factors made the group collectively proud of the team and the service they provide?

Concerns: What aspects of the service they provide were the most concerning?

Issues: Any other concerns they shared.
Members of the group represented expertise in each phase of the process, and a forum was established to encourage honesty and the sharing of issues without the fear of blame. The chemotherapy process was examined from referral to the completion of treatment. Hotspots were highlighted, and a proactive approach was taken for all issues related to chemotherapy.

What factors made the group collectively proud of the team and the service they provide?

- The morale of the staff is good and the service provided is praised by patients and relatives

- Staff felt that the actual location where chemotherapy is administered is of a high standard

- The environment where care is provided is pleasant and fit for its purpose

- Excellent information is available for patients

- There is an excellent multidisciplinary team spirit and open communication

What aspects of the service they provide were most concerning?

- Aspects of the chemotherapy prescription process

- The referral process from the multidisciplinary team is not always clear, and communication could be improved

- Staffing issues for therapy input meant that no cover is available on some days and this problem is exacerbated by short notices

- Prescription requests from the pharmacy are sometimes made on the same day as treatment. Medical staff are frequently too busy on the day of treatment to complete prescriptions, and notes are often unavailable. This increases the risk of a clinical incident and often means that patients have to wait for treatment

- At certain points in the chemotherapy process, communication is an issue

- Concerns were raised about drugs being administered without medical coverage

- Doctors frequently prescribe repeat chemotherapy without patient notes

- OPMAS (Oncology Patient Management Audit System) use is not maximized by all who have access and that includes doctors in clinics and nurses on the chemotherapy day unit

\section{Identified hand-offs and key issues}

- Hand-offs when the patient's care is transferred from one area to another individual or team

- Issues regarding the availability of beds for emergency patients 
- Consultants are often waiting up to two weeks for beds

- The number of review slots in clinics

- Staffing levels in all departments

- A lack of uniformity in the way consultants document the treatment plan

- Patients arriving for treatment with no prescription sheet

- No formalized structure for weighing patients on chemotherapy was in place

- Overbooking

- Concerns about capacity in the same-day unit

- Bank holidays

- Delays in obtaining blood tests before treatment

- The medical review process is not uniform

- Issues with the contribution of palliative care and social service teams

- Upon finishing therapy, no routine support group access

\section{Key measures for improvement}

Following discussion, it was evident that there were missing links and a lack of consistency between departments. As a result of the analysis, some recommendations were made, including the following:

a. There is a need for a better link between the medical staff, the pharmacy, and the chemotherapy day unit. The chemotherapy lead nurse has the role within the chemotherapy service to link patient's complaints with the chemotherapy process. Having fewer people involved reduces risk as well as developing the role of the chemotherapy services clerk whose role includes ensuring that prescriptions are organized and completed before the patient attends the day unit and ensuring that blood results are available before treatment is administered. That person would also act as a link between the different groups involved in the process and improve communication between the professionals involved in the process of chemotherapy treatment from first consultation with the doctor to the administration of the treatment. ${ }^{4-6}$

b. Benchmarking against other tertiary cancer centers to ascertain chemotherapy practices and to highlight best practices, taking into consideration local and national guidance.

c. Extending the usage of for information purposes and to improve communication.

d. Standard chemotherapy referral forms are completed by the oncologist who examined the patient. This includes which chemotherapy to use, the number of cycles, the frequency of blood tests and scans, and medical comments.

e. Weight forms. Patients are now being weighed in the chemotherapy day unit or outpatient departments. This only highlights a patient who has lost more than $10 \%$ of their original weight.

f. A patient information support group with contact numbers for patients who had previously received chemotherapy.

\section{Developments and outcomes achieved Referral form}

An intent to treat form was devised, and this form was tested using the Plan Do Study Act (PDSA) cycle. Initially, three consultants were involved in testing the feasibility of the introduced form. Gradually, the forms were introduced to the rest of the team and they are now used by all consultants and junior doctors in all clinics at the cancer center and peripheral clinics. The compliance of form use has been audited, and the results demonstrate a gradual increase in the use over the last few months from 84\% in January 2006 to $100 \%$ in May 2006. This high level of compliance has been maintained to date.

Having the treatment plan accessible to all staff has reduced errors, and Figure 1 demonstrates the improvements in the chemotherapy process as a result of the changes made in the practice.

In 2004, the number of incident reports (IR1s) was low, but upon reflection, this finding was due to the lack of practice reporting problems in the chemotherapy process by IR1s. As more problems were noticed in 2005, the trend shifted towards the department reporting chemotherapy problems so that they could be addressed. These problems included late prescriptions, dose reductions not noted in the records, and problems with chemotherapy prescriptions. The reduction of IR1s through the end of 2005 and 2006 reflected the improvement in the chemotherapy process achieved by the referral form, the better communication, and the systems set up by the Chemotherapy Improvement Group.

\section{Chemotherapy administration clerk}

This position was created to provide a missing link within the department. This new position has improved all the processes involved in chemotherapy administration. The chemotherapy administration clerk's roles include ensuring prescription requests are more organized and having blood 


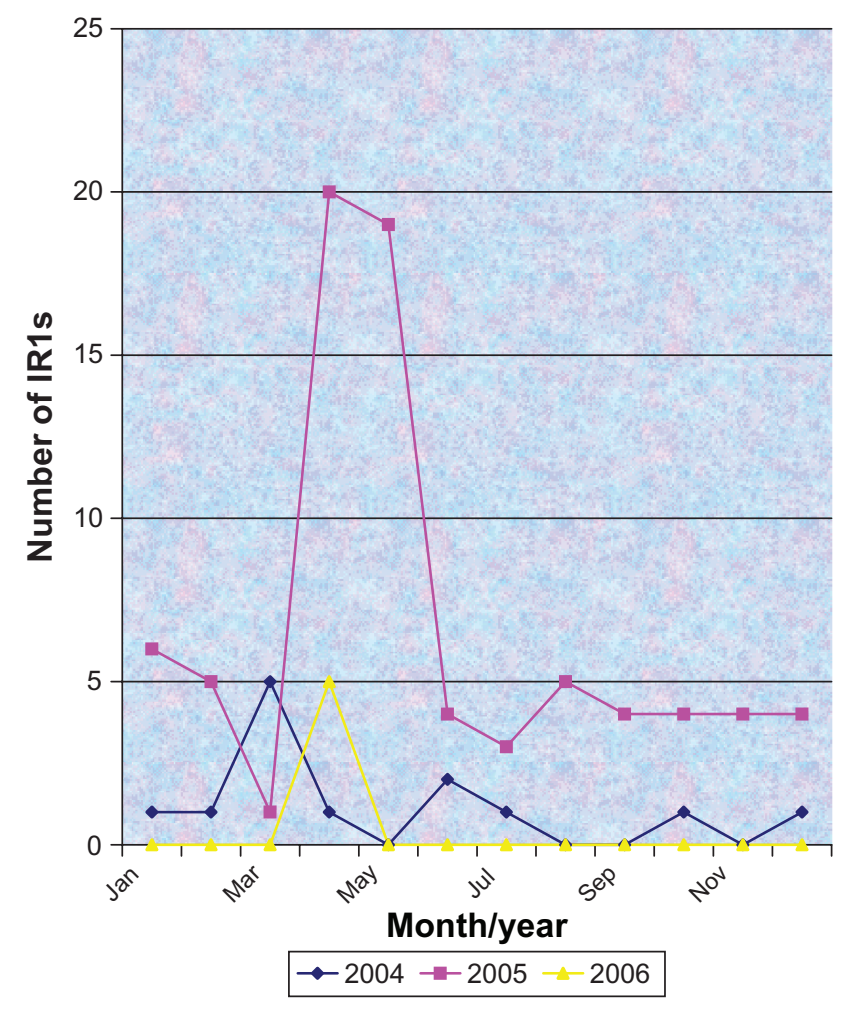

Figure I Improvements in the chemotherapy process.

results available the day before patients are treated, thereby enabling the preordering of chemotherapy, which reduces the wait times for patients. Having fewer people involved in the process ultimately reduces the risk throughout the chemotherapy process. In addition, communication among all departments has improved.

\section{Weight forms}

The weight forms were examined because patients were not being weighed at any particular time. This was flagged as a risk because the pharmacy was not being informed when patients had lost or gained $10 \%$ or more of their weight at their first treatment. Dose reductions were not performed due to this lack of information.

Subsequently, a decision by the group was made to weigh patients at every clinic or chemotherapy day-case unit visit if the patient had not been seen at the outpatient department. Every patient is now weighed at least once a month. The Trust weight chart was introduced and placed in the clinical notes section of the chart, and a process has been enabled to inform the appropriate departments when necessary. The pharmacy is contacted if a patient's weight changes by more than $10 \%$.

An audit of compliance with the weight forms in May 2006 was $85 \%$, which represented an increase of 5\% compared with a previous audit in March of the same year. This audit will be repeated again in April 2007 and will hopefully demonstrate a further increase in compliance.

\section{Patient information group}

It was highlighted by the staff and the patient representative that there is a lack of support for patients when their chemotherapy treatment has finished. The intention of this group was to produce a leaflet for patients for after treatment informing them of support groups and containing contact numbers. The Chemotherapy Improvement Group is also examining an "exit" assessment that incorporates the Chemotherapy Common Toxicity Criteria so the nurse can address any issues and refer to other specialists as appropriate.

\section{Conclusion}

The chemotherapy service is integral in the delivery of oncologic management at the North Wales Cancer Centre. When a potential risk was identified, it was essential to find the root cause and improve the system. By promoting a clear vision for the group and the identified structure (ie, Risk Management and Safer Patient Group), members were focused on the objectives. The risks were assessed, and the information was collated from all areas and disciplines involved in the process (patients, doctors, nurses, clerks, pharmacists, etc). By utilizing methods of modernization tools, several recommendations were made including the following:

The need for a link between medical staff, the pharmacy, and the chemotherapy day unit, a defined role of chemotherapy services clerk, and a standardized chemotherapy referral form.

Two new positions were developed to create a smoother process for chemotherapy treatment. The purpose of the chemotherapy services clerk is to bridge the gaps identified in the process. The other position incorporates the knowledge and skills of an experienced nurse by establishing a single focus for the division and examining the service development of different aspects for chemotherapy.

This group resulted in a sense of direction and knowledge about what we were aiming to achieve. These improvements in the service were a result of the multidisciplinary approach and commitment of the Chemotherapy Improvement Group, and although it was not the primary intention of this analysis, the implications of the Hospice care for these patients was also considered, but on further reflection, it was decided that this issue deserves separate consideration and it will be a theme of future research in our unit. 
We hope to use these findings to plan future studies looking into risk management issues in this group of patients.

\section{Disclosure}

No conflicts of interest were declared in relation to this paper.

\section{References}

1. CancerBACUP. Living with cancer: Waiting for treatment. London, UK: CancerBACUP; May 2004.

2. Department of Health. The NHS Cancer Plan. A plan for investment, a plan for reform. London, UK: Department of Health; 2000.
3. [NICE] National Institute for Clinical Excellence. Guidance on Cancer Services. Manual update. London, UK: NICE; May 2003.

4. Royal College of Radiologists. Principles to underpin the delivery of NHS Radiotherapy or Chemotherapy Services in Cancer units in the UK. 2006.

5. Summerhayes $M$. The impact of workload changes and staff availability on IV chemotherapy services. J Oncol Pharm Practice. 2003;9(4): 123-128.

6. General Medical Council. Good Medical Practice. London, UK: General Medical Council; 2006.
Risk Management and Healthcare Policy

\section{Publish your work in this journal}

Risk Management and Healthcare Policy is an international, peer-reviewed open access journal focusing on all aspects of public health, policy, and preventative measures to promote good health and improve morbidity and mortality in the population. The journal welcomes submitted papers covering original research, basic science, clinical \& epidemiological studies,

\section{Dovepress}

reviews and evaluations, guidelines, expert opinion and commentary, case reports and extended reports. The manuscript management system is completely online and includes a very quick and fair peer-review system, which is all easy to use. Visit http://www.dovepress.com/testimonials.php to read real quotes from published authors.

Submit your manuscript here: http://www.dovepress.com/risk-management-and-healthcare-policy-journal 